\title{
Service and Management for Multicast Based Audio/Video Collaboration System on CERNET
}

\author{
Xuan Zhang ${ }^{1,2}$, Xing $\mathrm{Li}^{1,2}$, and Qingguo Zhao ${ }^{1}$ \\ ${ }^{1}$ Network Research Center \\ ${ }^{2}$ Department of Electronic Engineering, \\ Tsinghua University ,Beijing China, 100084 \\ \{zhangx, xing\}@cernet.edu.cn, zhao-qg@mails.tsinghua.edu.cn
}

\begin{abstract}
Multicast based audio/video collaboration is one of representative applications in next generation internet. IP multicast has the advantage of saving bandwidth for group communication on large scale collaboration. The lack of ubiquitous multicast supporting and lack of effective service for multicast based A/V collaboration limit its application. This paper introduces the service and management of multicast based audio/video collaboration on China education and research network (CERNET). The multicast service, user and session management, performance monitoring and flow control are presented. The collaboration system has been applied on CERNET successfully.
\end{abstract}

Keywords: service, audio/video collaboration, multicast.

\section{Introduction}

Multi-party audio/video (A/V) collaboration systems are important applications in Next Generation Internet [1].In multi-party A/V collaboration, users could receive all group users' A/V stream with strong interactive characters. Among the solutions multicast based system may be ideal way for group collaboration. IP multicast could save bandwidth for many-to-many media communication, which makes it scalable. Some multicast based A/V systems as AccessGrid[2] have been employed recently. But for some reality reasons, the native multicast is not available ubiquitously, which confines the multicast applications extensively. On the other hand, the current $\mathrm{A} / \mathrm{V}$ collaboration system lacks effective service and management, for example, lack of guarantee on quality of service. In this paper, we introduce the service and management for multicast based A/V collaboration on CERNET. The IP multicast service on CERNET, user and session management, performance monitor and flow control are presented. We have implemented and applied the multicast A/V collaboration successfully.

\section{The Multicast Service on CERNET}

The native multicast is deployed at network level routers with multicast protocols. Now we have configured native multicast on CERNET backbone covering all the provincial capitals. The multicast routing protocol deployed on CERNET backbone is PIM-SM (Protocol Independent Multicast-Sparse Mode) [3]. The PIM-DM [4] is 
deployed in regional networks, and MSDP [5] are used for routing between different AS domains. In practice on deploying PIM-SM, we adopted 10 anycast RP(Rendezvous Point)s with same IP address via MSDP[5].

To users not in multicast domain we supply reflectors for them to join multicast group. Reflectors are application layer gateway which could convert unicast streaming data into multicast data and convert the multicast data into unicast data conversely.

Figure 1 shows the communication mechanism of reflector. The unicast users send their $\mathrm{A} / \mathrm{V}$ streaming to reflector, reflector receive the unicast data and retransmit them to multicast group. On the other hand, the multicast $\mathrm{A} / \mathrm{V}$ data from multicast users are received by reflector and resent to unicast users. By this way, users could receive all group users' $\mathrm{A} / \mathrm{V}$ streaming whether the users are in unicast or multicast domain.

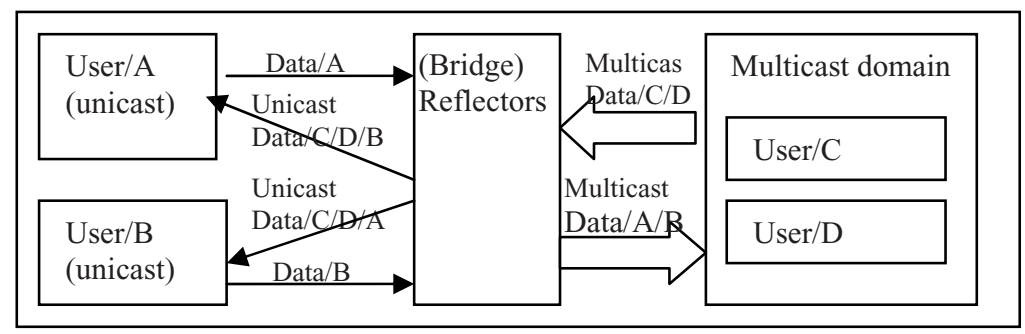

Fig . 1. The mechanism of reflector for unicast access

\section{User Management and Session Service}

We establish one website http://mvc.edu.cn[6] at CERNET national network center as collaboration service center for multicast $\mathrm{A} / \mathrm{V}$ collaboration (CSC-MVC).

\subsection{Users Manage and Venue Generation}

Users in $\mathrm{A} / \mathrm{V}$ collaboration could be classified into two types: chairman and participates. Chairman is the sponsor of collaboration venue. Commonly chairman applies for new venue and manages the venue. When chairman applies for create new venue, the Administrator of CSC-MVC would generate one new venue and add it to venuelist. The administrator assigned the session information for the venue.

Some authorities on venue manage may be endowed to the chairman by CSCMVC administrator . The chairman manages the participant users of this venue. If one user want to attend one venue, he must apply to the chairman or be invited by the chairman. In our service, the sponsor adds the invited users' name to the participants list, only the users in the invited participants list are permitted to enter the sponsor's venue room, otherwise he would be denied.

\subsection{Session Service}

Session information is created by CSC-MVC administrator. The session information mainly consists of IP/port pairs for audio/video. To users in native multicast domain, 
the multicast IP addresses are supplied, to users in unicast domain, the unicast IP addresses of reflectors are supplied.

The user first accesses the CSC-MVC website and login, authorized user could access venue room list (as figure 4 shows) and enter the prefer venue room if the user has been invited by chairman of this venue room. The session information stored in the venue is sent to end users when users enter venue. By parsing session information, the end user would get the multicast (and unicast) IP addresses/ports to launch their audio/video tools. Secret key and other session information are also used for launching audio/video. When at least two users enter the venue and communicate each other via audio/video tools, the multicast session is established. The session is terminated when all the users leave from the venues room. To temporary venue room, the CSC-MVC would delete the venue and take back the multicast IP /port pairs.

\section{Performance Monitor and Flow Control}

To guarantee the quality of multicast based $\mathrm{A} / \mathrm{V}$ collaboration, we design and implement the performance monitor and flow control scheme. We build a server for performance monitor and flow control charged by chairman. As Figure 2 shows, during collaboration the endpoints send their performance messages to server. The messages include the user's sending traffic, end-to-end delay and packet loss rate.

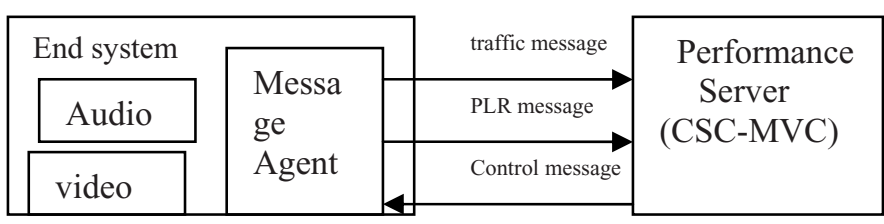

Fig .2. The performance monitor and flow control service

The end-to-end delay and PLR are calculated through RTP/RTCP protocols in audio/video tools. For example, PLR are calculated by received RTP packets. In a group with $\mathrm{N}$ users, each user can get N-1 PLR from other senders, figure 3 shows one user's end-to-end PLR report message. When all $\mathrm{N}$ users send their PLR message to the performance server, the server could get $\mathrm{N}^{*}(\mathrm{~N}-1)$ end-to-end PLRs. Chairman monitors the application performance from server. When congestion occurs, chairman would send rate control message to endpoints to adjust their sending bit rate. By this simple manual control, serious congestion could be avoided.

\begin{tabular}{|l|l|l|}
\hline Message Type & Seq_no & \multicolumn{2}{|c|}{ Timestamp } \\
\hline Local source & Local Cname(IP addr) & \\
\hline Source1 & Cname1 (IP addr) & PLR value \\
\hline Source2 & Cname2 (IP addr) & PLR value \\
\hline ...Source(i) & Cname(i)(IP addr) & PLR value \\
\hline
\end{tabular}

Fig .3. End user's end-to-end PLR message 


\section{Service and Application on CERNET}

The multicast based audio/video collaboration service has been provided on CERNET since 2002.In 2003 SARS period, the system advanced the A/V collaborations among domestic universities. Now the service has expanded to all provinces of China and become the A/V communication platform for CERNET members. Figure 4 shows one venue room list on collaboration service center for multicast $\mathrm{A} / \mathrm{V}$ collaboration.

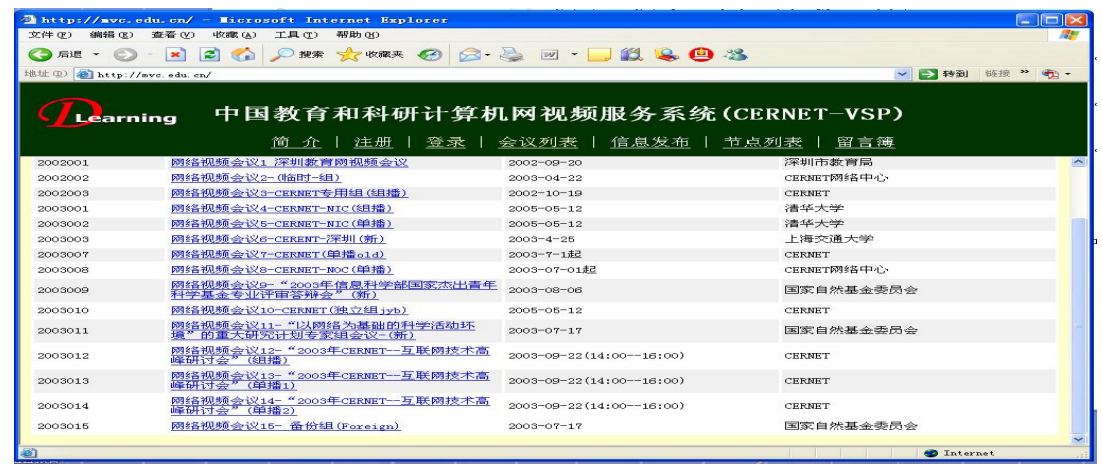

Fig .4. One venue room list on CERNET CSC-MVC from http://mvc.edu.cn

\section{Conclusions}

This paper introduces the service of multicast based audio/video collaboration systems on CERNET. The multicast service, users manage and session service, performance monitor and control are presented. The multicast based audio/video collaboration has been applied on CERNET successfully in nationwide.

\section{References}

[1] Internet2 consortium, http://dv.internet2.edu/ .

[2] Access Grid Project, http://www.accessgrid.org

[3] L. Wei, Protocol Independent Multicast-Sparse Mode (PIM-SM): Protocol Specification, Internet experimental RFC 2117, June (1997).

[4] Adams, J. Nicholas, W. Siadak, Protocol Independent Multicast - Dense Mode (PIM-DM): Protocol Specification (Revised), Internet experimental RFC 3973, January (2005).

[5] D. Kim, D. Meyer, H. Kilmer, D. Farinacci, Anycast Rendevous Point (RP) mechanism using Protocol Independent Multicast (PIM) and Multicast Source Discovery Protocol (MSDP), Internet informational RFC 3446, January (2003).

[6] Multicast based video service on CERNET, http://mvc.edu.cn . 